\title{
The mean ergodic theorem for nonexpansive mappings in multi-Banach spaces
}

\author{
Hassan Morghi Kenari ${ }^{1}$, Reza Saadati ${ }^{2}$ and Yeol Je Cho ${ }^{3^{*}}$
}

\author{
"Correspondence: yjcho@gnu.ac.kr \\ ${ }^{3}$ Department of Mathematics \\ Education and the RINS, \\ Gyeongsang National University, \\ Jinju, 660-701, Korea \\ Full list of author information is \\ available at the end of the article
}

\begin{abstract}
In this paper, we prove a mean ergodic theorem for nonexpansive mappings in multi-Banach spaces.
\end{abstract}

MSC: Primary 39A10; 39B72; secondary 47H10; 46B03

Keywords: ergodic theorem; nonexpansive mapping; multi-Banach space

\section{Introduction}

Let $X$ be a Banach space and $C$ be a closed convex subset of $X$. For each $j \geq 1$, a mapping $T_{j}: C \rightarrow C$ is said to be nonexpansive on $C$ if

$$
\left\|T_{j} x-T_{j} y\right\| \leq\|x-y\|
$$

for all $x, y \in C$. For each $j \geq 1$, let $F\left(T_{j}\right)$ be the set of fixed points of $T_{j}$. If $X$ is a strictly convex Banach space, then $F\left(T_{j}\right)$ is closed and convex.

In [1], Baillon proved the first nonlinear ergodic theorem such that, if $X$ is a real Hilbert space and $F\left(T_{j}\right) \neq \emptyset$ for each $j \geq 1$, then, for each $x \in C$, the sequence $\left\{S_{n, j} x\right\}$ defined by

$$
S_{n, j} x=\frac{1}{n}\left(x+T_{j} x+\cdots+T_{j}^{n-1} x\right)
$$

converges weakly to a fixed point of $T_{j}$. It was also shown by Pazy [2] that, if $X$ is a real Hilbert space and $S_{n, j} x$ converges weakly to $y \in C$, then $y \in F(T)$. These results were extended by Baillon [3], Bruck [4] and Reich [5, 6] and [7].

\section{Multi-Banach spaces}

The notion of a multi-normed space was introduced by Dales and Polyakov in [8]. This concept is somewhat similar to an operator sequence space and has some connections with the operator spaces and Banach lattices. Observations on multi-normed spaces and examples are given in [8-10].

Let $(E,\|\cdot\|)$ be a complex normed space and let $k \in \mathbb{N}$. We denote by $E^{k}$ the linear space $E \oplus \cdots \oplus E$ consisting of $k$-tuples $\left(x_{1}, \ldots, x_{k}\right)$, where $x_{1}, \ldots, x_{k} \in E$. The linear operations on $E^{k}$ are defined coordinate-wise. The zero element of either $E$ or $E^{k}$ is denoted by 0 . We denote by $\mathbb{N}_{k}$ the set $\{1,2, \ldots, k\}$ and by $\Sigma_{k}$ the group of permutations on $k$ symbols. 
Definition 2.1 A multi-norm on $\left\{E^{k}: k \in \mathbb{N}\right\}$ is a sequence $\left\{\|\cdot\|_{k}\right\}_{k \in \mathbb{N}}$ such that $\|\cdot\|_{k}$ is a norm on $E^{k}$ for each $k \in \mathbb{N}$ with $k \geq 2$ satisfying the following conditions:

(A1) $\left\|\left(x_{\sigma(1)}, \ldots, x_{\sigma(k)}\right)\right\|_{k}=\left\|\left(x_{1}, \ldots, x_{k}\right)\right\|_{k}\left(\sigma \in \Sigma_{k}, x_{1}, \ldots, x_{k} \in E\right)$;

(A2) $\left\|\left(\alpha_{1} x_{1}, \ldots, \alpha_{k} x_{k}\right)\right\|_{k} \leq\left(\max _{i \in \mathbb{N}_{k}}\left|\alpha_{i}\right|\right)\left\|\left(x_{1}, \ldots, x_{k}\right)\right\|_{k}\left(\alpha_{1}, \ldots, \alpha_{k} \in \mathbb{C}, x_{1}, \ldots, x_{k} \in E\right)$;

(A3) $\left\|\left(x_{1}, \ldots, x_{k-1}, 0\right)\right\|_{k}=\left\|\left(x_{1}, \ldots, x_{k-1}\right)\right\|_{k-1}\left(x_{1}, \ldots, x_{k-1} \in E\right)$;

(A4) $\left\|\left(x_{1}, \ldots, x_{k-1}, x_{k-1}\right)\right\|_{k}=\left\|\left(x_{1}, \ldots, x_{k-1}\right)\right\|_{k-1}\left(x_{1}, \ldots, x_{k-1} \in E\right)$.

In this case, we say that $\left\{\left(E^{k},\|\cdot\|_{k}\right)\right\}_{k \in \mathbb{N}}$ is a multi-normed space.

Lemma $2.2([10])$ Suppose that $\left\{\left(E^{k},\|\cdot\|_{k}\right)\right\}_{k \in \mathbb{N}}$ is a multi-normed space and take $k \in \mathbb{N}$. Then we have the following:

(1) $\|(x, \ldots, x)\|_{k}=\|x\|(x \in E)$;

(2) $\max _{i \in \mathbb{N}_{k}}\left\|x_{i}\right\| \leq\left\|x_{1}, \ldots, x_{k}\right\|_{k} \leq \sum_{i=1}^{k}\left\|x_{i}\right\| \leq k \max _{i \in \mathbb{N}_{k}}\left\|x_{i}\right\|\left(x_{1}, \ldots, x_{k} \in E\right)$.

It follows from (2) that, if $(E,\|\cdot\|)$ is a Banach space, then $\left(E^{k},\|\cdot\|_{k}\right)$ is a Banach space for each $k \in \mathbb{N}$. In this case $\left\{\left(E^{k},\|\cdot\|_{k}\right)\right\}_{k \in \mathbb{N}}$ is a multi-Banach space.

Now, we give two important examples of multi-norms for an arbitrary normed space $E[8]$.

Example 2.3 The sequence $\left\{\|\cdot\|_{k}\right\}_{k \in \mathbb{N}}$ on $\left\{E^{k}: k \in \mathbb{N}\right\}$ defined by

$$
\left\|\left(x_{1}, \ldots, x_{k}\right)\right\|_{k}:=\max _{i \in \mathbb{N}_{k}}\left\|x_{i}\right\| \quad\left(x_{1}, \ldots, x_{k} \in E\right)
$$

is a multi-norm, which is called the minimum multi-norm. The terminology 'minimum' is justified by the property (2).

Example 2.4 Let $\left\{\left(\|\cdot\|_{k}^{\alpha}: k \in \mathbb{N}\right): \alpha \in A\right\}$ be the (nonempty) family of all multi-norms on $\left\{E^{k}: k \in \mathbb{N}\right\}$. For each $k \in \mathbb{N}$, set

$$
\left\|\left(x_{1}, \ldots, x_{k}\right)\right\|_{k}:=\sup _{\alpha \in A}\left\|\left(x_{1}, \ldots, x_{k}\right)\right\|_{k}^{\alpha} \quad\left(x_{1}, \ldots, x_{k} \in E\right)
$$

Then $\left\{\|\cdot\|_{k}\right\}_{k \in \mathbb{N}}$ is a multi-norm on $\left\{E^{k}: k \in \mathbb{N}\right\}$, which is called the maximum multi-norm.

We need the following observation, which can easily be deduced from the triangle inequality for the norm $\|\cdot\|_{k}$ and the property (2) of multi-norms.

Lemma 2.5 Suppose that $k \in \mathbb{N}$ and $\left(x_{1}, \ldots, x_{k}\right) \in E^{k}$. For each $j \in\{1, \ldots, k\}$, let $\left\{x_{n}^{j}\right\}_{n \geq 1}$ be a sequence in $E$ such that $\lim _{n \rightarrow \infty} x_{n}^{j}=x_{j}$. Then, for each $\left(y_{1}, \ldots, y_{k}\right) \in E^{k}$, we have

$$
\lim _{n \rightarrow \infty}\left(x_{n}^{1}-y_{1}, \ldots, x_{n}^{k}-y_{k}\right)=\left(x_{1}-y_{1}, \ldots, x_{k}-y_{k}\right)
$$

Definition 2.6 Let $\left\{\left(E^{k},\|\cdots\|_{k}\right)\right\}_{k \in \mathbb{N}}$ be a multi-normed space. A sequence $\left\{x_{n}\right\}_{n \geq 1}$ in $E$ is called a multi-null sequence if, for any $\varepsilon>0$, there exists $n_{0} \in \mathbb{N}$ such that

$$
\sup _{k \in \mathbb{N}}\left\|\left(x_{n}, \ldots, x_{n+k-1}\right)\right\|_{k}<\varepsilon \quad\left(n \geq n_{0}\right) .
$$


Let $x \in E$. We say that the sequence $\left\{x_{n}\right\}_{n \geq 1}$ is multi-convergent to a point $x \in E$ and write

$$
\lim _{n \rightarrow \infty} x_{n}=x
$$

if $\left\{x_{n}-x\right\}_{n}$ is a multi-null sequence.

\section{Main results}

To prove the main results in this paper, first, we introduce some lemmas.

Lemma 3.1 ([11]) Let $\left\{\left(X^{j},\|\cdot\|_{j}\right)\right\}_{j \in \mathbb{N}}$ be a uniformly convex multi-Banach space with modulus of the convexity $\delta$. Let $x_{j}, y_{j} \in X$. If $\left\|\left(x_{1}, \ldots, x_{j}\right)\right\|_{j} \leq r,\left\|\left(y_{1}, \ldots, y_{j}\right)\right\|_{j} \leq r, r \leq R$ and $\left\|\left(x_{1}-y_{1}, \ldots, x_{j}-y_{j}\right)\right\|_{j} \geq \epsilon>0$, then

$$
\left\|\left(\lambda x_{1}+(1-\lambda) y_{1}, \ldots, \lambda x_{j}+(1-\lambda) y_{j}\right)\right\|_{j} \leq r\left(1-2 \lambda(1-\lambda) \delta_{R}(\epsilon)\right)
$$

for all $\lambda \in[0,1]$, where $\delta_{R}(\epsilon)=\delta\left(\frac{\epsilon}{R}\right)$.

To proceed, let $\left\{\left(X^{j},\|\cdot\|_{j}\right)\right\}_{j \in \mathbb{N}}$ denote a uniformly convex multi-Banach space with modulus of the convexity $\delta$.

Lemma 3.2 Let $C$ be a closed convex subset of $X$ and for each $j \geq 1, T_{j}: C \rightarrow C$ be a nonexpansive mapping. Let $x \in C, f_{j} \in F\left(T_{j}\right)$ for each $j \geq 1$ and $0<\alpha \leq \beta<1$. Then, for any $\epsilon>0$, there exists $N>0$ such that, for all $n \geq N$,

$$
\begin{aligned}
& \|\left(T_{1}^{k}\left(\lambda T_{1}^{n} x+(1-\lambda) f_{1}\right)-\left(\lambda T_{1}^{n+k} x+(1-\lambda) f_{1}\right),\right. \\
& \left.\quad \ldots, T_{j}^{k}\left(\lambda T_{j}^{n} x+(1-\lambda) f_{j}\right)-\left(\lambda T_{j}^{n+k} x+(1-\lambda) f_{j}\right)\right) \|_{j} \\
& \quad<\epsilon
\end{aligned}
$$

for all $k>0$ and $\lambda \in[\alpha, \beta]$.

Proof Put

$$
\begin{aligned}
& r=\lim _{n}\left\|\left(T_{1}^{n} x-f_{1}, \ldots, T_{j}^{n} x-f_{j}\right)\right\|_{j}, \quad R=\left\|\left(x-f_{1}, \ldots, x-f_{j}\right)\right\|_{j}, \\
& c=\min \{2 \lambda(1-\lambda): \alpha \leq \lambda \leq \beta\} .
\end{aligned}
$$

For given $\epsilon>0$, choose $d>0$ such that $\frac{r}{r+d}>1-c \delta_{R}(\epsilon)$. Then there exists $N>0$ such that, for all $n \geq N$,

$$
\left\|\left(T_{1}^{n} x-f_{1}, \ldots, T_{j}^{n} x-f_{j}\right)\right\|_{j}<r+d
$$

For each $n \geq N, k>0$ and $\alpha \leq \lambda \leq \beta$, we put

$$
u_{j}=(1-\lambda)\left(T_{j}^{k} z-f_{j}\right), \quad v_{j}=\lambda\left(T_{j}^{n+k} x-T_{j}^{k} z\right),
$$

where $z_{j}=\lambda T_{j}^{n} x+(1-\lambda) f_{j}$. Then we have

$$
\left\|\left(u_{1}, \ldots, u_{j}\right)\right\|_{j} \leq \lambda(1-\lambda)\left\|\left(T_{1}^{n} x-f_{1}, \ldots, T_{j}^{n} x-f_{j}\right)\right\|_{j}
$$


and

$$
\left\|\left(v_{1}, \ldots, v_{j}\right)\right\|_{j} \leq \lambda(1-\lambda)\left\|\left(T_{1}^{n} x-f_{1}, \ldots, T_{j}^{n} x-f_{j}\right)\right\|_{j} .
$$

Suppose that

$$
\begin{aligned}
& \left\|\left(u_{1}-v_{1}, \ldots, u_{j}-v_{j}\right)\right\|_{j} \\
& \quad=\left\|\left(T_{1}^{k} z-\left(\lambda T_{1}^{n+k} x+(1-\lambda) f_{1}\right), \ldots, T_{1}^{k} z-\left(\lambda T_{j}^{n+k} x+(1-\lambda) f_{j}\right)\right)\right\|_{j} \\
& \quad \geq \epsilon .
\end{aligned}
$$

Then, by Lemma 3.1, we have

$$
\begin{aligned}
& \left\|\left(\lambda u_{1}+(1-\lambda) v_{1}, \ldots, \lambda u_{j}+(1-\lambda) v_{j}\right)\right\|_{j} \\
& \quad=\lambda(1-\lambda)\left\|\left(T_{1}^{n+k} x-f_{1}, \ldots, T_{j}^{n+k} x-f_{j}\right)\right\|_{j} \\
& \quad \leq \lambda(1-\lambda)\left\|\left(T_{1}^{n} x-f_{1}, \ldots, T_{j}^{n} x-f_{j}\right)\right\|_{j}\left(1-2 \lambda(1-\lambda) \delta_{R}(\epsilon)\right) \\
& \quad \leq \lambda(1-\lambda)\left\|\left(T_{1}^{n} x-f_{1}, \ldots, T_{j}^{n} x-f_{j}\right)\right\|_{j}\left(1-c \delta_{R}(\epsilon)\right) .
\end{aligned}
$$

Hence we have

$$
(r+d)\left(1-c \delta_{R}(\epsilon)\right)<r \leq(r+d)\left(1-C \delta_{R}(\epsilon)\right)
$$

which is a contradiction. This completes the proof.

Lemma 3.3 (Browder [12]) Let $C$ be a closed convex subset of $X$ and $T_{j}: C \rightarrow C$ be a nonexpansive mapping. If $\left\{u_{i}\right\}$ is a weakly convergent sequence in $C$ with the weak limit $u_{0}$ and $\lim _{i}\left\|u_{i}-T_{j} u_{i}\right\|=0$, then $u_{0}$ is a fixed point of $T_{j}$.

Lemma 3.4 Let $C$ be a closed convex subset of $X$ and, for each $j \geq 1, T_{j}: C \rightarrow C$ be a nonexpansive mapping. Then, for all $x \in C$ and $n>0$,

$$
\lim _{i \rightarrow \infty} \sup _{j \rightarrow \infty}\left\|\left(T_{1}^{k} S_{n, 1} T_{1}^{i} x-S_{n, 1} T_{1}^{k} T_{1}^{i} x, \ldots, T_{j}^{k} S_{n, j} T_{j}^{i} x-S_{n, j} T_{j}^{k} T_{j}^{i} x\right)\right\|_{j}=0
$$

uniformly for each $k \geq 1$.

Proof By induction on $n$, we prove this lemma. First, we prove the conclusion in the case $n=2$. Put

$$
\begin{aligned}
& r=\lim _{n \rightarrow \infty} \sup _{j \geq 1}\left\|\left(T_{1}^{n+1} x-T_{1}^{n} x, \ldots, T_{j}^{n+1} x-T_{j}^{n} x\right)\right\|_{j}, \\
& R=\left\|\left(x-T_{1} x, \ldots, x-T_{j} x\right)\right\|_{j}, \quad x_{i, j}=T_{j}^{i} x
\end{aligned}
$$

for each $i \geq 1$. 
If $r \neq 0$, then, for any $\epsilon>0$, choose $c>0$ such that $\frac{r}{r+c}>1-\delta_{R}(\epsilon) / 2$. Then there exists $N>0$ such that, for all $i \geq N$,

$$
\left\|\left(T_{1}^{k} x_{i, 1}-T_{1}^{k+1} x_{i, 1}, \ldots, T_{j}^{k} x_{i, j}-T_{j}^{k+1} x_{i, j}\right)\right\|_{j} \leq r+c
$$

for each $k \geq 1$. If we put

$$
u_{j}=\frac{1}{2}\left(T_{j}^{k} z-T_{j}^{k} x_{i, j}\right), \quad v_{j}=\frac{1}{2}\left(T_{j}^{k+1} x_{i, j}-T_{j}^{k} z_{j}\right),
$$

where $i \geq N, k>0$ and $z_{j}=\frac{1}{2}\left(x_{i, j}+T_{j} x_{i, j}\right)$, then we have

$$
\begin{aligned}
\left\|\left(u_{1}, \ldots, u_{j}\right)\right\|_{j} & \leq \frac{1}{2}\left\|\left(z_{1}-x_{i, 1}-z_{j}-x_{i, j}\right)\right\|_{j} \\
& =\frac{1}{4}\left\|\left(T_{1} x_{i, 1}-x_{i, 1}, \ldots, T_{j} x_{i, j}-x_{i, j}\right)\right\|_{j} \\
& \leq \frac{1}{4}(r+c) .
\end{aligned}
$$

Similarly, we have $\left\|\left(v_{1}, \ldots, v_{j}\right)\right\|_{j} \leq \frac{1}{4}(r+c)$. Suppose that

$$
\begin{aligned}
& \left\|\left(u_{1}-v_{1}, \ldots, u_{j}-v_{j}\right)\right\|_{j} \\
& \quad=\left\|\left(T_{1}^{k} z_{1}-\frac{1}{2}\left(T_{1}^{k+1} x_{i, 1}+T_{1}^{k} x_{i, 1}\right), \ldots, T_{j}^{k} z_{j}-\frac{1}{2}\left(T_{j}^{k+1} x_{i, j}+T_{j}^{k} x_{i, j}\right)\right)\right\|_{j} \\
& \quad \geq \epsilon
\end{aligned}
$$

Then, by Lemma 3.1, we have

$$
\begin{aligned}
\left\|\frac{1}{2}\left(u_{1}+v_{1}, \ldots, u_{j}+v_{j}\right)\right\|_{j} & =\frac{1}{4}\left\|\left(T_{1}^{k+1} x_{i, 1}-T_{1}^{k} x_{i, 1}, \ldots, T_{j}^{k+1} x_{i, j}-T_{j}^{k} x_{i, j}\right)\right\|_{j} \\
& \leq \frac{1}{4}(r+c)\left(1-\frac{1}{2} \delta_{R}(\epsilon)\right)
\end{aligned}
$$

which contradicts $r>(r+c)\left(1-\frac{1}{2} \delta_{R}(\epsilon)\right)$.

If $r=0$, then, for any $\epsilon>0$, choose $i>0$ so large that $\sup _{j}\left\|\left(u_{1}, \ldots, u_{j}\right)\right\|_{j}<\frac{\epsilon}{2}$. Hence we have

$$
\begin{aligned}
\sup _{j \geq 1}\left\|\left(T_{1}^{k} z_{1}-\frac{1}{2}\left(T_{1}^{k+1} x_{i, 1}+T_{1}^{k} x_{i, 1}\right), \ldots, T_{j}^{k} z_{j}-\frac{1}{2}\left(T_{j}^{k+1} x_{i, j}+T_{j}^{k} x_{i, j}\right)\right)\right\|_{j} \\
\quad=\sup _{j \geq 1}\left\|\left(u_{1}-v_{1}, \ldots, u_{j}-v_{j}\right)\right\|_{j} \\
\quad \leq \sup _{j \geq 1}\left\|\left(u_{1}, \ldots, u_{j}\right)\right\|_{j}+\sup _{j \geq 1}\left\|\left(v_{1}, \ldots, v_{j}\right)\right\|_{j} \\
\quad<\epsilon .
\end{aligned}
$$

This completes the proof of the case $n=2$. 
Now, suppose that

$$
\lim _{i \rightarrow \infty} \sup _{j \geq 1}\left\|\left(T_{1}^{k} S_{n-1,1} x_{i, 1}-S_{n-1,1} T_{1}^{k} x_{i, 1}, \ldots, T_{j}^{k} S_{n-1, j} x_{i, j}-S_{n-1, j} T_{j}^{k} x_{i, j}\right)\right\|_{j}=0
$$

uniformly for each $k \geq 1$. We claim that

$$
\lim _{i \rightarrow \infty} \sup _{j \geq 1}\left\|\left(S_{n-1,1} T_{1} x_{i, 1}-x_{i, 1}, \ldots, S_{n-1, j} T_{j} x_{i, j}-x_{i, j}\right)\right\|_{j}
$$

exists. Put

$$
r=\liminf _{i \rightarrow \infty} \sup _{j \geq 1}\left\|\left(S_{n-1,1} T_{1} x_{i, 1}-x_{i, 1}, \ldots, S_{n-1, j} T_{j} x_{i, j}-x_{i, j}\right)\right\|_{j} .
$$

For any $\epsilon>0$, choose $i>0$ such that

$$
\sup _{j \geq 1}\left\|\left(S_{n-1,1} T_{1} x_{i, 1}-x_{i, 1}, \ldots, S_{n-1, j} T_{j} x_{i, j}-x_{i, j}\right)\right\|_{j}<r+\frac{\epsilon}{2}
$$

and

$$
\sup _{j \geq 1}\left\|\left(S_{n-1,1} T_{1}^{k} x_{i+1,1}-T_{1}^{k} S_{n-1,1} x_{i+1,1}, \ldots, S_{n-1, j} T_{j}^{k} x_{i+1, j}-T_{j}^{k} S_{n-1, j} x_{i+1, j}\right)\right\|_{j}<\frac{\epsilon}{2} .
$$

Then we have

$$
\begin{aligned}
& \sup _{j \geq 1}\left\|\left(S_{n-1,1} T_{1} x_{i+k, 1}-x_{i+k, 1}, \ldots, S_{n-1, j} T_{j} x_{i+k, j}-x_{i+k, j}\right)\right\|_{j} \\
& \leq \sup _{j \geq 1}\left\|\left(S_{n-1,1} T_{1}^{k} x_{i+1,1}-T_{1}^{k} S_{n-1,1} x_{i+1,1}, \ldots, S_{n-1, j} T_{j}^{k} x_{i+1, j}-T_{j}^{k} S_{n-1, j} x_{i+1, j}\right)\right\|_{j} \\
& \quad+\sup _{j \geq 1}\left\|\left(T_{1}^{k} S_{n-1,1} x_{i+1,1}-T_{1}^{k} x_{i, 1}, \ldots, T_{j}^{k} S_{n-1, j} x_{i+1, j}-T_{j}^{k} x_{i, j}\right)\right\|_{j} \\
& \quad<\frac{\epsilon}{2}+r+\frac{\epsilon}{2} \\
& =r+\epsilon
\end{aligned}
$$

for all $k \geq 1$. Therefore, we have

$$
\begin{aligned}
& \limsup _{i \rightarrow \infty} \sup _{j \geq 1}\left\|\left(S_{n-1,1} T_{1} x_{i, 1}-x_{i, 1}, \ldots, S_{n-1, j} T_{j} x_{i, j}-x_{i, j}\right)\right\|_{j} \\
& \quad=\limsup _{k \rightarrow \infty} \sup _{j \geq 1}\left\|\left(S_{n-1,1} T_{1} x_{i+k, 1}-x_{i+k, 1}, \ldots, S_{n-1, j} T_{j} x_{i+k, j}-x_{i+k, j}\right)\right\|_{j} \\
& \quad<r+\epsilon .
\end{aligned}
$$

Since $\epsilon>0$ is arbitrary, we have

$$
\begin{aligned}
& \limsup _{i \rightarrow \infty} \sup _{j \geq 1}\left\|\left(S_{n-1,1} T_{1} x_{i, 1}-x_{i, 1}, \ldots, S_{n-1, j} T_{j} x_{i, 1}-x_{i, 1}\right)\right\|_{j} \\
& \quad \leq \liminf _{i \rightarrow \infty} \sup _{j \geq 1}\left\|\left(S_{n-1,1} T_{1} x_{i, 1}-x_{i, 1}, \ldots, S_{n-1, j} T_{j} x_{i, j}-x_{i, j}\right)\right\|_{j},
\end{aligned}
$$

i.e., $\lim _{i \rightarrow \infty} \sup _{j \geq 1}\left\|\left(S_{n-1,1} T_{1} x_{i, 1}-x_{i, 1}, \ldots, S_{n-1, j} T_{j} x_{i, j}-x_{i, j}\right)\right\|_{j}$ exists. 
Now, we put

$$
r=\lim _{i \rightarrow \infty} \sup _{j \geq 1}\left\|\left(S_{n-1,1} T_{1} x_{i, 1}-x_{i, 1}, \ldots, S_{n-1, j} T_{j} x_{i, j}-x_{i, j}\right)\right\|_{j}
$$

If $r \neq 0$, then, for any $\epsilon$, choose $c>0$ such that

$$
\frac{r-c}{r+2 c}>1-\left(2 \frac{(n-1)}{n^{2}}\right) \delta_{3 r}(\epsilon) .
$$

Then there exists $N>0$ such that, if, for all $i \geq N$, we put

$$
u_{j}=\frac{n}{(n-1)}\left(T_{j}^{k} S_{n, j} x_{i, j}-T_{j}^{k} x_{i, j}\right), \quad v_{j}=n\left(S_{n-1, j} T_{j}^{k} x_{i+1, j}-T_{j}^{k} S_{n, j} x_{i, j}\right),
$$

so

$$
\begin{aligned}
& \left\|\left(u_{1}, \ldots, u_{j}\right)\right\|_{j} \leq\left\|\left(S_{n-1,1} T_{1} x_{i, 1}-x_{i, 1}, \ldots, S_{n-1, j} T_{j} x_{i, j}-x_{i, j}\right)\right\|_{j} \leq r+c, \\
& \left\|\left(v_{1}, \ldots, v_{2}\right)\right\|_{j} \\
& \leq n\left\|\left(S_{n-1,1} T_{1}^{k} x_{i+1,1}-T_{1}^{k} S_{n-1,1} x_{i+1,1}, \ldots, S_{n-1, j} T_{j}^{k} x_{i+1, j}-T_{j}^{k} S_{n-1, j} x_{i+1, j}\right)\right\|_{j} \\
& +\left\|\left(S_{n-1,1} T_{1} x_{i, 1}-x_{i, 1}, \ldots, S_{n-1, j} T_{j} x_{i, j}-x_{i, j}\right)\right\|_{j} \\
& \leq r+2 c
\end{aligned}
$$

and

$$
\begin{aligned}
& \left\|\left(u_{1}-v_{1}, \ldots, u_{j}-v_{j}\right)\right\|_{j} \\
& \quad=\frac{n}{n-1}\left\|\left(T_{1}^{k} S_{n, 1} x_{i, 1}-S_{n, 1} T_{1}^{k} x_{i, 1}, \ldots, T_{j}^{k} S_{n, j} x_{i, j}-S_{n, j} T_{j}^{k} x_{i, j}\right)\right\|_{j} .
\end{aligned}
$$

Hence, by the method in the proof of the case $n=2$, we have

$$
\sup _{j \geq 1}\left\|\left(T_{1}^{k} S_{n, 1} x_{i, 1}-S_{n, 1} T_{1}^{k} x_{i, 1}, \ldots, T_{j}^{k} S_{n, j} x_{i, j}-S_{n, j} T_{j}^{k} x_{i, j}\right)\right\|_{j}<\epsilon
$$

for all $k \geq 1$ and $i \geq N$.

If $r=0$, then, as in the proof of the case $n=2$, there exists $N^{\prime}$ such that, for each $i \geq N^{\prime}$,

$$
\sup _{j \geq 1}\left\|\left(u_{1}, \ldots, u_{j}\right)\right\|_{j}<\frac{\epsilon}{2}, \quad \sup _{j \geq 1}\left\|\left(v_{1}, \ldots, v_{j}\right)\right\|_{j}<\frac{\epsilon}{2} .
$$

Therefore, we have

$$
\sup _{j \geq 1}\left\|\left(T_{1}^{k} S_{n, 1} x_{i, 1}-S_{n, 1} T_{1}^{k} x_{i, 1}, \ldots, T_{j}^{k} S_{n, j} x_{i, j}-S_{n, j} T_{j}^{k} x_{i, j}\right)\right\|_{j}<\epsilon
$$

This completes the proof.

Now, assume that the norm of $X$ is Frechet differentiable and then we have the following. 
Proposition $3.5([4,6,13])$ Let $C$ be a closed convex subset of $X$ and, for each $j \geq 1, T_{j}$ : $C \rightarrow C$ be a nonexpansive mapping. If we put $W_{j}(x)=\cap_{m} \overline{c o}\left\{T_{j}^{k} x: k \geq m\right\}$ for all $x \in C$, then $W_{j}(x) \cap F\left(T_{j}\right)$ is at most one point.

In this paper, we give a new proof of the following theorem, which is due to Reich [6].

Theorem 3.6 Let $\left\{\left(X^{j},\|\cdot\|_{j}\right)\right\}_{j \in \mathbb{N}}$ be a uniformly convex multi-Banach space which has the Fréchet differentiable norm. Let $C$ be a closed convex subset of $X$ and, for each $j \geq 1$, $T_{j}: C \rightarrow C$ be a nonexpansive mapping. Then the following statements are equivalent:

(1) $F\left(T_{j}\right) \neq \emptyset$.

(2) $\left\{T_{j}^{n} x\right\}$ is bounded for all $x \in C$.

(3) For all $x \in C,\left\{S_{n} T_{j}^{i} x\right\}$ converges weakly to a point $\left(y_{1}, \ldots, y_{j}\right) \in C^{j}$ uniformly for each $i \geq 1$.

Proof $(1) \Longleftrightarrow(2)$ is well known in [12].

(3) $\Longleftrightarrow(2)$ Suppose that, for some $x \in C$, there exists an unbounded subsequence $\left\{T_{j}^{n_{i}} x\right\}$ of $\left\{T_{j}^{n} x\right\}$. For each $j \geq 1$, since $T_{j}$ is a nonexpansive mapping, it follows that, for each $m>0$, the sequence $\left\{S_{m<j} T_{j}^{n_{i}} x\right\}$ is also unbounded, which contradicts the condition (3).

(2) $\Longleftrightarrow(3)$ Since $\left\{T_{j}^{n} x\right\}$ is bounded and

$$
\begin{aligned}
\| & \left(T_{1} S_{n, 1} T_{1}^{i} x-S_{n, 1} T_{1}^{i} x, \ldots, T_{j} S_{n, j} T_{j}^{i} x-S_{n, j} T_{j}^{i} x\right) \|_{j} \\
\leq & \left\|\left(T_{1} S_{n, 1} T_{1}^{i} x-S_{n, 1} T_{1} T_{1}^{i} x, \ldots, T_{j} S_{n, j} T_{j}^{i} x-S_{n, j} T_{j} T_{j}^{i} x\right)\right\|_{j} \\
& +\left\|\left(S_{n, 1} T_{1} T_{1}^{i} x-S_{n, 1} T_{1}^{i} x, \ldots, S_{n, j} T_{j} T_{j}^{i} x-S_{n, j} T_{j}^{i} x\right)\right\|_{j} \\
\leq & \left\|\left(T_{1} S_{n, 1} T_{1}^{i} x-S_{n, 1} T_{1} T_{1}^{i} x, \ldots, T_{j} S_{n, j} T_{j}^{i} x-S_{n, j} T_{j} T_{j}^{i} x\right)\right\|_{j} \\
& +\frac{1}{n}\left\|\left(T_{1}^{i+1+n} x-T_{1}^{i} x, \ldots, T_{j}^{i+1+n} x-T_{j}^{i} x\right)\right\|_{j},
\end{aligned}
$$

there exists a sequence $\left\{S_{n, j} T_{j}^{i_{n}} x\right\}$ such that

$$
\lim _{n \rightarrow \infty} \sup _{j \geq 1}\left\|\left(T_{1} S_{n, 1} T_{1}^{i_{n}} x-S_{n, 1} T_{1}^{i_{n}} x, \ldots, T_{j} S_{n, j} T_{j}^{i_{n}} x-S_{n, j} T_{j}^{i_{n}} x\right)\right\|_{j}=0
$$

Then, by Lemma 3.3 and Proposition 3.5, it follows that any weakly multi-convergent subsequence of $\left\{S_{n, j} T_{j}^{i_{n}} x\right\}$ multi-converges weakly to a point $y_{j}$, i.e., $S_{n, j} T_{j}^{i_{n}} x \rightarrow y_{j}$, where $y_{j}=W_{j}(x) \cap F\left(T_{j}\right)$. Also, by Lemma 3.4, it follows that

$$
\lim _{n \rightarrow \infty} \sup _{j \geq 1}\left\|\left(T_{1} S_{n, 1} T_{1}^{i_{n}+k n+i} x-S_{n, 1} T_{1}^{i_{n}+k n+i} x, \ldots, T_{j} S_{n, j} T_{j}^{i_{n}+k n+i} x-S_{n, j} T_{j}^{i_{n}+k n+i} x\right)\right\|_{j}=0
$$

for all $i, k \geq 1$. Therefore, $S_{n, j} T_{j}^{i_{n}+k n} x_{i} \rightarrow y_{j}$ uniformly for each $k \geq 1$.

On the other hand, for each $n \geq 1$ with $m \geq i_{n}$, we have

$$
\begin{aligned}
S_{m, j} T_{j}^{i} x & =\frac{1}{m} \sum_{k=0}^{m-1} T_{j}^{k} x_{i} \\
& =\frac{1}{m}\left(\sum_{k=i_{n}+t n}^{m-1} T_{j}^{k} x_{i}+n\left(\sum_{k=0}^{t} S_{n} T_{j}^{i_{n}+k n} x_{i}\right)+\sum_{k=0}^{i_{n}} T_{j}^{k} x_{i}\right),
\end{aligned}
$$


where $m=t n+i_{n}+r, r<n$. Since $\left\{S_{n, j} T_{j}^{i_{n}+k n} x_{i}\right\}$ multi-converges to $y_{j}$ uniformly for each $k \geq 1$, it follows that $\left\{S_{m, j} T_{j}^{i} x\right\}$ converges weakly to $y_{j}$ uniformly for each $i \geq 1$. This completes the proof.

\section{Competing interests}

The authors declare that they have no competing interests.

\section{Authors' contributions}

All authors carried out the proof. All authors conceived of the study, and participated in its design and coordination. All authors read and approved the final manuscript.

\section{Author details}

'Department of Mathematics, Science and Research Branch, Islamic Azad University, Ashrafi Esfahani Ave, Tehran, 14778 , Iran. ${ }^{2}$ Department of Mathematics and Computer Science, Iran University of Science and Technology, Tehran, Iran.

${ }^{3}$ Department of Mathematics Education and the RINS, Gyeongsang National University, Jinju, 660-701, Korea.

\section{Acknowledgements}

The third author was supported by the Basic Science Research Program through the National Research Foundation of Korea (NRF) funded by the Ministry of Education, Science and Technology (Grant Number: 2011-0021821).

Received: 11 April 2014 Accepted: 13 June 2014 Published: 22 July 2014

\section{References}

1. Baillon, JB: Un theoreme de type ergodique pour les contractions non lineaires dans un espace de Hilbert. C. R. Acad. Sci. Paris Sér. A-B 280, 1511-1514 (1975)

2. Pazy, A: On the asymptotic behavior of iterates of nonexpansive mappings in Hilbert space. Isr. J. Math. 26, 197-204 (1977)

3. Baillon, JB: Comportement asymptotique des itérés de contractions non linéaires dans les espaces $L_{p}$. C. R. Acad. Sci. Paris Sér. A-B 286, 157-159 (1978)

4. Bruk, RE: A simple proof of the mean ergodic theorem for nonlinear contractions in Banach space. Isr. J. Math. 32, 107-116 (1979)

5. Reich, S: Almost convergence and nonlinear ergodic theorems. J. Approx. Theory 24, 269-272 (1978)

6. Reich, S: Weak convergence theorems for nonexpansive mappings in Banach space. J. Math. Anal. Appl. 67, 274-276 (1979)

7. Reich, S: Nonlinear ergodic theory in Banach space. Argonne National Laboratory Report \# $79-69$ (1979)

8. Dales, HG, Polyakov, ME: Multi-normed spaces and multi-Banach algebras. Preprint

9. Dales, HG, Moslehian, MS: Stability of mappings on multi-normed spaces. Glasg. Math. J. 49, 321-332 (2007)

10. Moslehian, MS, Nikodem, K, Popa, D: Asymptotic aspect of the quadratic functional equation in multi-normed spaces. J. Math. Anal. Appl. 355, 717-724 (2009)

11. Groetsch, CW: A note on segmention Mann iterates. J. Math. Anal. Appl. 40, 369-372 (1972)

12. Browder, FE: Nonlinear Operators and Nonlinear Equations of Evolution in Banach Space. Pure Math., vol. 18. Am. Math. Soc., Providence (1976)

13. Hirano, N: A proof of the mean ergodic theorem for nonexpansive mappings in Banach space. Proc. Am. Math. Soc 78, 361-365 (1980)

doi:10.1186/1029-242X-2014-259

Cite this article as: Kenari et al.: The mean ergodic theorem for nonexpansive mappings in multi-Banach spaces. Journal of Inequalities and Applications 2014 2014:259.

\section{Submit your manuscript to a SpringerOpen ${ }^{\odot}$ journal and benefit from:}

- Convenient online submission

- Rigorous peer review

- Immediate publication on acceptance

- Open access: articles freely available online

- High visibility within the field

- Retaining the copyright to your article 